\title{
Biodegradable lignin nanocontainers $\dagger$
}

Cite this: RSC Adv., 2014, 4, 11661

\author{
Doungporn Yiamsawas, ${ }^{a}$ Grit Baier, ${ }^{a}$ Eckhard Thines, ${ }^{\text {bc }}$ Katharina Landfester ${ }^{a}$ \\ and Frederik R. Wurm ${ }^{\star a}$
}

Received 25th December 2013

Accepted 5th February 2014

DOI: $10.1039 / c 3 r a 47971 d$

www.rsc.org/advances

The abundant biomaterial lignin was used to prepare hollow nanocapsules by interfacial polyaddition in inverse miniemulsions. These cross-linked lignin nanocontainers can be loaded with hydrophilic substances which can be released by an enzymatic trigger from natural plant extracts revealing them as potential nanocontainers for agricultural applications.

Lignin is one of the most abundant renewable biomaterials which can be extracted from plants and which represents approximately $15-30 \%$ of their total mass besides cellulose. ${ }^{1}$ In spite of these high amounts and its ready availability, lignin is normally considered as a non-preferred byproduct from the paper industry for example with over 30 mio tons per annum. ${ }^{2} \mathrm{~A}$ major reason for this is probably the complexity of the lignin structure with very broad molecular weight distributions and limited solubility, thus only $2 \%$ of lignin (mainly lignosulfonates) are applied in industry or in agricultural uses as binders for animal feed pellets, bricks, ceramics, or road dust, dispersants for oil well drilling products etc. ${ }^{1,3,4}$ The main structural elements of lignin, a phenolic polymer, are three basic structural elements (4-hydroxyphenyl, guaiacyl, and syringyl residues) which are connected via aromatic and aliphatic ether bonds that build up a hyperbranched, i.e. irregularly branched polymer. ${ }^{1,2}$ Because lignin has both phenolic and aliphatic hydroxyl groups, its further modification is obvious and was investigated in various publications. Recently, lignin doped with multi-walled carbon nanotubes were studied as chemical

\footnotetext{
${ }^{a}$ Max-Planck-Institut für Polymerforschung, Ackermannweg 10, 55128 Mainz, Germany.E-mail: wurm@mpip-mainz.mpg.de

${ }^{b} I B W F$ e.V., Institute of Biotechnology and Drug Research, Erwin-Schrödinger-Str. 56, 67663 Kaiserslautern, Germany

'Institute of Biotechnology, Johannes Gutenberg-University, Duesbergweg 10-14, 55128 Mainz, Germany

$\dagger$ Electronic supplementary information (ESI) available: Synthetic details, additional spectra and electron microscopy images, additional degradation studies. See DOI: 10.1039/c3ra47971d
}

sensors. ${ }^{5}$ In addition, lignin films were modified at the $\mathrm{OH}^{-}$ groups to grow polymer brushes. ${ }^{6}$

Herein, we make use of a water-soluble lignin fraction to generate lignin-polyurea/polyurethane nanocontainers at the interface of stable water nanodroplets in an inverse miniemulsion. With this protocol, potentially biodegradable nanocontainers with an aqueous core are obtained allowing the efficient encapsulation of hydrophilic substances as reported for other systems previously. ${ }^{7,8}$ As lignin is biodegradable and nontoxic, this platform can be widely applied as nanocontainers for the encapsulation of bioactive drugs, for example, to generate advanced agricultural applications or to encapsulate fertilizers or pesticides which was only studied on the macroscopic scale (i.e. blending) to date..$^{9-11}$

This is the first report on the utilization of the highly abundant biomaterial lignin to generate hollow nanocontainers that can be cleaved by naturally occurring enzymes. In an inverse miniemulsion the crosslinking polymerization of lignin was tailored to take place only at the interface of stable aqueous nanodroplets (acting as capsule templates) dispersed in an organic solvent. ${ }^{12}$ Enzyme-responsive, cleavable cross-linked lignin nanocontainers were thus generated by selective polyaddition at the oil-water interface. We chose the reaction of toluene diisocyanate (TDI) with the lignin hydroxyl groups to generate a cross-linked shell surrounding a liquid aqueous core (Scheme 1).

Lignosulfonic acid sodium salt or lignin was dissolved together with sodium chloride in Milli-Q water in order to generate the dispersed phase which was then mixed with cyclohexane containing the biocompatible surfactant polyglycerol polyricinoleate (PGPR). The pre-emulsion was stirred at room temperature and subsequently ultrasonicated in order to generate the stable miniemulsion. The polyaddition reaction at the interface of the miniemulsion nanodroplets was initiated after a solution of TDI in cyclohexane was added drop-wise to the previously formed miniemulsion (compare Scheme 1). The reaction was kept at room temperature overnight in order to ensure complete consumption of the active species. The 


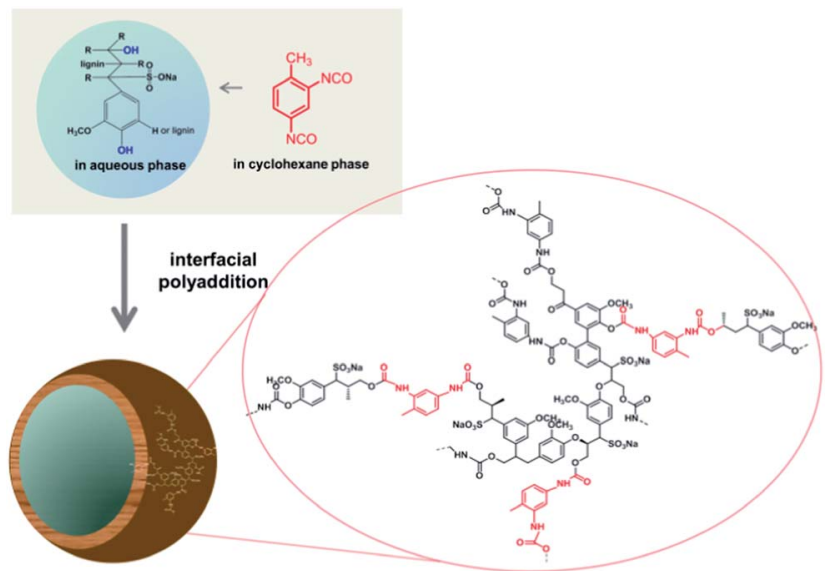

Scheme 1 Synthetic protocol for the generation of hollow lignin nanocontainers by inverse miniemulsion.

obtained lignin nanocapsule dispersion in cyclohexane was stable over a period of several months. The diameters of the capsules and the morphology were determined via dynamic light scattering (DLS) and electron microscopy (SEM and TEM), respectively. The nanocontainers were then transferred into aqueous dispersion without the use of an additional surfactant due to the presence of sulfonic acid groups of the starting lignosulfonic acid producing (after dialysis) a surfactant free aqueous dispersion of the lignin nanocapsules. For lignin alkali (which does not carry additional ionic side groups) the same synthetic protocol was applied; however in this case a surfactant (sodium dodecylsulfate) had to be added to allow redispersion of the nanocapsules in water.

Since the isocyanate groups of TDI at the water-oil interface not only react with the hydroxyl group in the lignin molecules but can also be hydrolyzed by water to amine groups, also urea linkages can be found in the capsule shell, consequently pol$y$ (urea-urethane) cross-linked lignin nanocontainers are generated (Scheme 1). From the IR spectra the presence of the urethane carbonyl bond at $1715 \mathrm{~cm}^{-1}$ and the urea carbonyl vibration at $1639 \mathrm{~cm}^{-1}$ were confirmed as presented in Fig. 1.

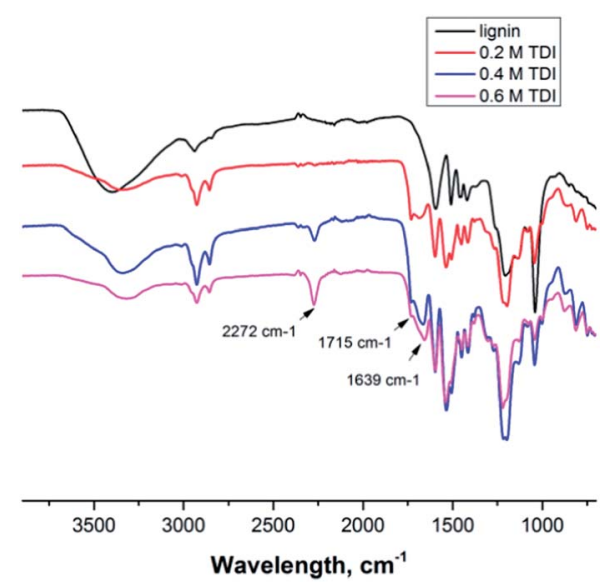

Fig. 1 FT-IR spectra of lignin (top) and several lignin nanocontainers with varying amount of cross-linker from dispersion in cyclohexane.
Moreover with increasing the amount of TDI in the reaction, the intensity of these vibrations increases indicating a higher degree of crosslinking within the nanocapsule shell. Further, from the FT-IR spectra, the ratio between urethane and urea bonds can be estimated by the peak area of the characteristic band of urethane/urea which decreased from 0.9 to 0.6 with increasing amount of TDI. This can be rationalized by pronounced reaction of the isocyanate groups with water in the presence of a large excess of TDI resulting in urea formation. ${ }^{13,14}$ All prepared nanocontainers are highly functional: in cyclohexane dispersion the presence of isocyanate groups on the capsule surface can also be detected (at $2272 \mathrm{~cm}^{-1}$ ) which could be used to further functionalize the capsules from the outside.

These reactive groups are hydrolyzed to amine groups when the nanocontainers are dispersed in water which could be also addressed in further reactions (compare ESI Fig. S2 $\dagger$ ).

The cross-linked lignin nanocontainers exhibit diameters in the range of 162 to $220 \mathrm{~nm}$ and 311 to 390 in cyclohexane or water, respectively, as determined by DLS with varying amount of the cross-linker TDI (compare Table S1 and Fig. S1 in the ESI $\dagger$ ). The nanocontainers remained stable after being redispersed in water over a period of several weeks. Nevertheless the diameter of the nanocapsules in buffer solution is slightly larger than in cyclohexane probably due to swelling and some aggregation. To further confirm the morphology of the lignin nanocapsules, scanning and transmission electron microscopy techniques were performed as shown in Fig. 2 for the cyclohexane dispersions (a representative image for the redispersed nanocontainers can be found in the ESI Fig. S3 $\uparrow$ ). In all cases hollow capsule structures were identified and their diameters correspond well to the result from dynamic light scattering. From the TEM images an average capsule wall thickness for all dry samples of $10-20 \mathrm{~nm}$ can be determined (compare ESI Fig. S4†).

Laccases are well-known enzymes present in the lignolitic systems of many fungi. Such enzymes are typically used for delignification, so in order to proof natural nanocapsules degradation, laccase is used as a natural model to degrade the lignin capsules. White rot fungi have been discussed in large

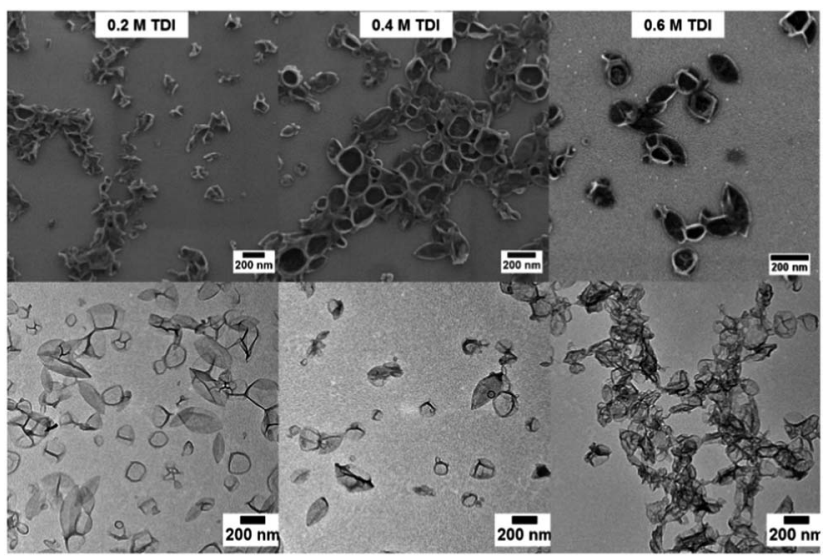

Fig. 2 SEM (top) and TEM (bottom) images of several hollow lignin nanocontainers with varying amount of cross-linker. 


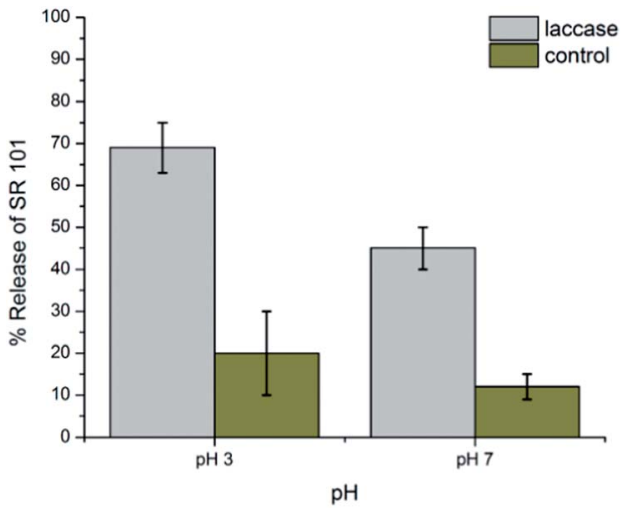

Fig. 3 Release profile of the lignin nanocontainers prepared with $0.2 \mathrm{mM}$ of TDI after degraded by Xylaria sp. IBWF A55-2009 laccase at $50^{\circ} \mathrm{C}$.

detail as natural resources for laccases. In contrast to the lignolitic enzymes of these basidiomycetes, ascomycetes of the Xylariaceae family have not been intensively been studied. However, the presence of Xylaria species on decaying wood in almost all forests indicates an important role in the recycling of lignin. ${ }^{15}$ In order to investigate the biodegradability of the lignin nanocapsules, the hydrophilic fluorescent dye sulforhodamine (SR101) was encapsulated, which is released upon degradation of the capsule shell. The cleavage of the cross-linked lignin shell was studied by a laccase from the fungus Xylaria sp. IBWF-A552009. Fig. 3 shows the release of SR101 for the enzymatic cleavage by laccase (IBWF-A55-2009 laccase) at $50{ }^{\circ} \mathrm{C}$ after incubation over a period of $24 \mathrm{~h}$ at $\mathrm{pH} 3$ and $\mathrm{pH}$ 7. At $\mathrm{pH} 3$ and $50{ }^{\circ} \mathrm{C}$, which are the optimum working conditions for this enzyme, a high amount of released SR101 was detected; however, also at neutral $\mathrm{pH}$ enzymatic release was proven to be effective. The enzyme extraction also proved to be efficient in cleaving the capsule shell and the released amount depends on the cross-linking density (compare ESI Fig. S5-S7†). This indicates that the lignin nanocontainers with higher degree of crosslinking have a denser network with polyurea-urethane bond are probably cleaved much slower by the enzyme.

In addition, the release profile of the dye at room temperature at $\mathrm{pH} 7$ which was assumed as the most natural condition was also studied and similar results were detected (compare ESI $\dagger$ ). However, the rate of the dye release in natural conditions was slower than in the proper condition of enzyme. These experiments prove that the polyurethane/urea linkages do not hamper the enzymatic cleavage of the novel lignin nanocapsules and make them useful nanocontainers for future agricultural applications.

\section{Conclusions}

In conclusion, this work presents the first synthesis of hollow lignin nanocontainers with an aqueous core. They have been prepared by selective polyaddition at the interface of stable aqueous nanodroplets in an inverse miniemulsion. The reaction setup allows the efficient encapsulation of hydrophilic substances (drugs, fertilizers, pesticides) which was proved by the hydrophilic model compound (SR101). The obtained lignin nanocontainers showed diameters in the range of 150-200 nm and were stable in organic or aqueous dispersion over a period of several weeks-months. In addition, enzymatic degradation of the lignin nanocontainers has been performed by laccase as a model enzyme and also by a mixed enzyme cocktail extracted from natural fungi. This robust protocol makes the degradable lignin nanocontainers to an attractive long-term release system based on the renewable resource lignin that is currently under investigation in our group.

\section{Notes and references}

1 F. G. Calvo-Flores and J. A. Dobado, ChemSusChem, 2010, 3, 1227.

2 H. Hatakeyama and T. Hatakeyama, in Biopolymers: Lignin, Proteins, Bioactive Nanocomposites, ed. A. Abe, K. Dusek and S. Kobayashi, Springer-Verlag Berlin, Berlin, 2010, vol. 232, pp. 1-63.

3 D. Stewart, Ind. Crops Prod., 2008, 27, 202.

4 J. H. Lora and W. G. Glasser, J. Polym. Environ., 2002, 10, 39.

5 F. A. C. Faria, D. V. Evtuguin, A. Rudnitskaya, M. T. S. R. Gomes, J. A. B. P. Oliveira, M. P. F. Graca and L. C. Costa, Polym. Int., 2012, 61, 788.

6 G. Gao, J. I. Dallmeyer and J. F. Kadla, Biomacromolecules, 2012, 13, 3602.

7 F. Tiarks, K. Landfester and M. Antonietti, Langmuir, 2001, 17, 908.

$8 \mathrm{~K}$. Landfester and C. K. Weiss, in Modern Techniques for Nano- and Microreactors/-Reactions, ed. F. Caruso, 2010, vol. 229, pp. 1-49.

9 M. Fernandez-Perez, M. Villafranca-Sanchez, F. FloresCespedes and I. Daza-Fernandez, Carbohydr. Polym., 2011, 83, 1672.

10 F. Flores-Céspedes, C. I. Figueredo-Flores, I. DazaFernández, F. Vidal-Peña, M. Villafranca-Sánchez and M. Fernández-Pérez, J. Agric. Food Chem., 2012, 60, 1042.

11 D. Ciolacu, A. M. Oprea, N. Anghel, G. Cazacu and M. Cazacu, Mater. Sci. Eng., C, 2012, 32, 452.

12 K. Malzahn, F. Marsico, K. Koynov, K. Landfester, C. K. Weiss and F. R. Wurm, ACS Macro Lett., 2014, 3, 40-43.

13 E.-M. Rosenbauer, M. Wagner, A. Musyanovych and K. Landfester, Macromolecules, 2010, 43, 5083.

14 G. Baier, A. Musyanovych, M. Dass, S. Theisinger and K. Landfester, Biomacromolecules, 2010, 11, 960.

15 D. H. Nghi, B. Bittner, H. Kellner, N. Jehmlich, R. Ullrich, M. J. Pecyna, P. Nousiainen, J. Sipilä, M. Huong le, M. Hofrichter and C. Liers, Appl. Environ. Microbiol., 2012, 78, 4893. 